\section{RMD Open}

Rheumatic \&

Musculoskeletal Diseases

\title{
Hydroxychloroquine and the risk of respiratory infections among $R A$ patients
}

Joel M Kremer (D), ${ }^{1}$ George Reed, ${ }^{2,3}$ Dimitrios A Pappas, ${ }^{4}$ LR Harold, ${ }^{5}$ Kevin Kane, ${ }^{6}$ Jeffrey Greenberg, ${ }^{7}$ Kevin Winthrop ${ }^{8}$

To cite: Kremer JM, Reed G, Pappas DA, et al. Hydroxychloroquine and the risk of respiratory infections among RA patients. RMD Open 2020;6: e001389. doi:10.1136/ rmdopen-2020-001389

Received 9 July 2020 Revised 18 September 2020 Accepted 17 October 2020
Check for updates

(c) Author(s) (or their employer(s)) 2020. Re-use permitted under CC BY-NC. No commercial re-use. See rights and permissions. Published by BMJ.

For numbered affiliations see end of article.

\section{Correspondence to} Joel M Kremer; JKremer@corrona.org

\section{ABSTRACT}

Objectives To determine the effect of hydroxychloroquine on the incidence of new respiratory infections in a large registry of rheumatoid arthritis (RA) patients compared with a matched cohort receiving other conventional diseasemodifying antirheumatic drugs (csDMARDs).

Methods We reviewed physician-reported infections including upper respiratory infections (URI), bronchitis and pneumonia in the Corrona RA registry from June 2008 to February 2020 with the goal of comparing infections in biologic/targeted synthetic (b/ts) DMARDs naive HCQ starts compared with starts of other csDMARDs and no HCQ. Patients on different interventions were compared using time-varying adjusted Cox models adjusting for age, sex, duration of RA, BMI, disease activity, smoking status, concurrent medications, season of the year, year of onset and history of serious infections, diabetes or cardiovascular disease (CVD). A secondary analysis in a set of propensitymatched starts were also compared adjusting for timevarying covariates. The analysis was repeated including URI and bronchitis only and also for serious respiratory infections only.

Results No evidence of differences was found in the incidence of any respiratory infection (URI, bronchitis, pneumonia) in patients receiving $\mathrm{HCQ}$ compared with other CSDMARDs: HR=0.87 (0.70 to1.07) in adjusted analyses and $\mathrm{HR}=0.90$ (0.70 to 1.17) in adjusted matched analysis. Similar results were found in the analysis of URI and bronchitis only and for serious respiratory infections only. Conclusions In patients with RA, the risk for respiratory infections was similar among patients using $\mathrm{HCQ}$ as compared to other non-biologic DMARDs.

\section{INTRODUCTION}

Because of the recent SARS-CoV-2 pandemic, there has been speculation in the scientific and lay press regarding the potential benefit of either chloroquine or hydroxychloroquine (HCQ) in protecting from COVID-19 either by decreasing the incidence, severity or duration of the infection. A small clinical study in France was reported to be associated with diminished viral load, ${ }^{1}$ while a subsequent study did not replicate

\section{Key messages}

- The effect of hydroxychloroquine on the prevention of viral upper respiratory infections is not known.

- This study showed that there is no benefit for prevention of upper respiratory infectionsfor patients with rheumatoid arthritis on hydroxychloroquine.

- Given the speculation of the potential anti-viral effect of hydroxychloroquine for SARS-CoV2, the finding of an absence of a protective effect for other respiratory infections suggests that it would be unlikely if the drug had a specific protective effect for this virus if the effect does not exist for other viral infections in this population.

a positive effect. ${ }^{2}$ In addition, an in vitro study has demonstrated that perhaps HCQ was associated with diminished viral uptake and replication, ${ }^{3}$ while a subsequent investigation suggested an effective therapeutic dose based on in vitro data. ${ }^{4}$ Non-peerreviewed results of small RCTs have conflicting findings. ${ }^{5} 6$

Perhaps because of these data, there are now reports of widely diminished availability of HCQ in pharmacies. Both rheumatoid arthritis (RA) and systemic lupus erythematosus (SLE) patients have legitimate concerns associated with difficulty accessing this medication that they need in order to maintain disease control. Indeed, pharmaceutical companies have committed to step up their production of HCQ given the speculation of possible benefit and the perception of minimal downside. ${ }^{7}$

Given the recent speculation regarding the possible protective effects of HCQ in SARS CoV-2 infections ${ }^{8}$ and the subsequent clinical questions regarding whether individuals 
currently using HCQ have a lower risk of such infections, understanding the baseline risk of respiratory infections in general among HCQ users is of utmost relevance and importance. The understanding of the effects of HCQ on routine community-acquired viral infections will be informative as the drug is investigated for a possible protective effect on COVID-19 in a population-based fashion. It is also reasonable to hypothesise that if there is a measurable HCQ antiviral effect then patients on chronic therapy with the drug may experience less frequent respiratory infections typically caused by viruses. Accordingly, we chose to use the Corrona registry to evaluate the risk of respiratory infections among HCQ users as compared to those using other non-biologic DMARDs.

\section{MATERIALS AND METHODS}

\section{Patient population}

The Corrona RA registry, collecting data since 2001, includes data derived from a network of academic $(13 \%)$ and community rheumatologists $(87 \%)$ as has previously been described. ${ }^{9}$ All patients are consented for participation using the New England Institutional Review Board. Data are gathered at clinical visits from both the patient and the treating provider including detailed safety and treatment information. As part of the routine data collected at the time of a clinical encounter, the registry mandates active collection of safety data including both historical and contemporaneous information on infections including upper respiratory infections (URIs), bronchitis and pneumonia. The type of infection is collected including whether intravenous antibiotics were administered and whether the infection led to hospitalisation, recovery or death. For this analysis, the first start of HCQ by a patient was included and the first start of other csDMARDs by a patient was included if they occurred after June 2008 since the specific indicator for bronchitis was added at that time. Patients could contribute more than one start but not of the same csDMARD. Patients did not have to be HCQ naïve or other csDMARD naive at the time of the start of the csDMARD. All eligible starts had to occur in b/tsDMARD naïve RA patients and have at least one month of follow-up after the start. If the order of a start and an outcome of interest were indeterminate, the start was excluded from analysis.

Eligible medication starts were divided into two cohorts: the hydroxychloroquine (HCQ) cohort and csDMARD cohort (with no HCQ use) (figure 1).

\section{Outcomes}

Safety data, including infections, are collected at the time of the registry visit and include all interval occurrences since the prior registry visit. The primary outcome was the combined incidence of URI, bronchitis and pneumonia. Secondary outcomes were the combined incidence of URI and bronchitis and the incidence of serious respiratory infections (URIs, bronchitis and pneumonia only).

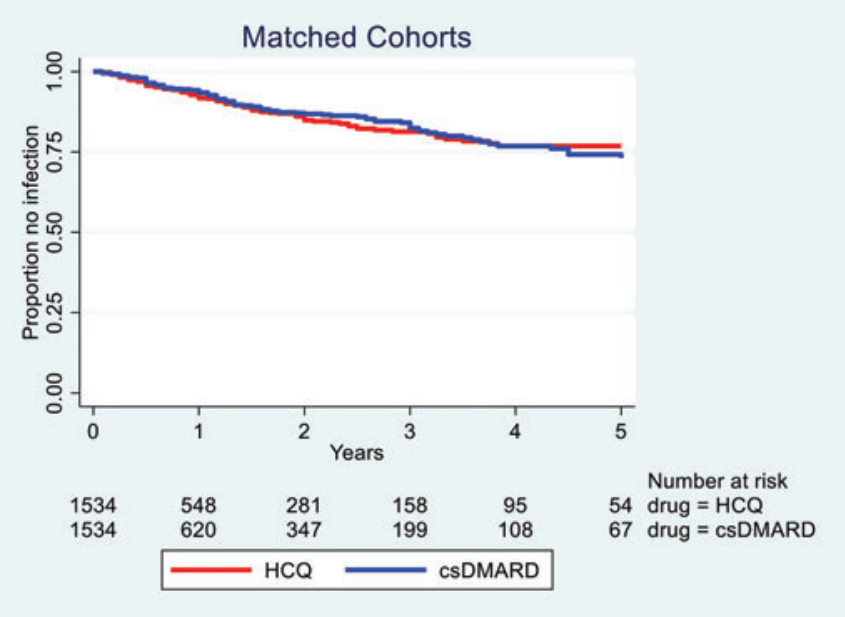

Figure 1 Starts included based on eligibility.

Serious infections are cases that led to hospitalisation or needing intravenous antibiotics for therapy. Incident infections and the date of the infection (month/year) are captured on the physician (MD) form at the time of a registry visit when patients are also specifically questioned about infections that may have occurred in the interim period between visits. Serious infections are also captured by targeted adverse event (TAE) forms between clinical visits. MD form and TAE reported serious infections are combined and assessed to avoid duplicate reporting of serious infections.

\section{Exposure time}

The HCQ cohort consisted of HCQ starts possibly added to other csDMARDs but without simultaneous concurrent csDMARDs starts. Follow-up (exposure time) continued until the occurrence of either of the following events:

- Respiratory infection of interest (URI, bronchitis, pneumonia; URI, bronchitis; serious infection).

- Discontinuation of HCQ +30 days.

- Start of a b/tsDMARD +30 days.

The csDMARD cohort consisted of non-HCQ csDMARD starts. Follow-up time continued until the earliest of these events:

- Respiratory infection of interest (URI, bronchitis, pneumonia; URI, bronchitis; serious infection).

- Discontinuation of the index csDMARD +30 days.

- Start of a b/tsDMARD +30 days.

- Start of HCQ.

Patients could contribute more than one start but could only contribute one start for any single csDMARD (eg, a patient could contribute one HCQ start, one MTX start and one leflunomide start)

\section{Analysis}

Comparison of patient and clinical characteristics at the index date-start of HCQ or non-HCQ csDMARD—were compared using t-test for continuous variables and $\chi^{2}$ test for categorical variables. 
Unadjusted Kaplan-Meier curves were estimated for the HCQ and csDMARD cohorts. Cox regression models estimated HRs (hazard of respiratory infection in HCQ starters vs csDMARD starters) adjusted for a priori selected baseline characteristics (sex, age, duration of RA, body mass index (BMI), year of start, history of serious infections) and time-varying covariates (disease activity as measured by the Clinical Disease Activity Index (CDAI)), prior use of 1 versus 2 or more csDMARDs, use of prednisone and its dose, season of the year, history of diabetes and history of cardiovascular disease (CVD). Time-varying covariates were updated at each Corrona clinical visit. Covariance estimates were adjusted for clustering by patient (some patients contribute more than one start). ${ }^{8}$ Proportional hazard assumptions were tested and not rejected. Values in the manuscript are presented as mean (SD).

A secondary sensitivity analysis was carried out using a population of matched HCQ starters with csDMARD starters. A propensity for HCQ start versus csDMARD start score was estimated using a logistic regression model using the following selected variables: gender, age, CDAI, prednisone use, duration of RA, number of csDMARDs at start, history of serious infections, diabetes mellitus, CVD and BMI. HCQ starts were matched 1:1 with csDMARD starts with a caliper of 0.01. Unadjusted Kaplan-Meier curves were estimated in the matched populations and a Cox regression model estimated the HR of risk of infection in HCQ to csDMARD matched starters using time-varying covariates and a covariance estimate adjusted for clustering by pair.

The analyses were repeated for all three outcomes.

\section{RESULTS}

Figure 1 illustrates the starts included based on eligibility. For the primary analysis of URI, Bronchitis and Pneumonia as the outcome, there were a total of 6792 starts from 5726 patients. There were 2270 starts of HCQ, and 4422 starts of csDMARDs other than HCQ (2903 starts on MTX, 965 on leflunomide, 600 on azulfidine, 35 on azathioprine, 18 on minocycline and 1 ciclosporin). Since the total starts were depending on the outcome considered (see figure 1), counts differed slightly for the two other outcomes. In the matched starts for the primary outcome, there were $1534 \mathrm{HCQ}$ starts and 1534 csDMARD starts (831 MTX, 385 leflunomide, 290 azulfidine, 16 azathioprine, 11 minocycline and 1 ciclosporin). At baseline, disease activity was greater in patients on nonHCQ csDMARDs (clinical disease activity index (CDAI) of 17.2 (13.8) and 12.7 (10.8) respectively, $\mathrm{p}<00183.001)$. A prior history of serious infection was more common in patients on HCQ than those on the other csDMARDs $(\mathrm{p}=0.041) \quad$ (table 1$)$. A significantly greater percentage of patients were on $>7.5 \mathrm{mg}$ of prednisone at the time of start in the csDMARD cohort versus HCQ $(14.1 \%$ vs $7.3 \%$, respectively, $\mathrm{p}<0.001$ ) but a greater number of $\mathrm{HCQ}$ patients were on 2 or more csDMARDs at the time of start compared with the other cohort $(52.3 \%$ vs $14.2 \%$, respectively, $\mathrm{p}<0.001)$. None of these differences remained significant after matching (table 1 ). The number of starts were a little higher (20-30 additional starts) for the secondary outcomes since there were fewer events and thus fewer exclusions (figure 1). Similar differences existed for secondary outcomes with no differences in the matched cohorts.

There were 543 respiratory infections of which 234 were reported as URI, 184 as bronchitis and 125 as pneumonia. Table 2 lists estimates of overall rates of respiratory infections per 100 patient-years and by cohort. The estimated HRsand 95\% CIs comparing risk in HCQ versus csDMARD starts of combined URI, bronchitis and pneumonia; combined URI and bronchitis; and serious infections are provided in table 2. While the HR estimates are slightly less than 1, CIs extend for most estimates above 1.1 .

The HR comparing risk in HCQ versus csDMARD was $0.87(0.70,1.07)$ and $0.90(0.70,1.17)$ for the total respiratory infections of URI, bronchitis and pneumonia from the adjusted model for all starters and the adjusted model for matched starters, respectively. HR estimates and CIs were similar when comparing only URI and bronchitis as the outcomes (adjusted HR of 0.85 (0.68 to1.08) and 0.93 (0.70 to 1.25) for all starters and matched starters, respectively) or when comparing serious respiratory infections HR of 1.29 (0.76 to 2.18) and 1.10 (0.58 to 2.12) (table 2). In the matched starters, a Kaplan-Meier curve for the primary outcome of URI, bronchitis and pneumonia illustrates overlapping proportion with no infection (unadjusted for time-varying covariates) (figure 2).

\section{DISCUSSION}

In summary, we found little evidence to suggest that the use of HCQ, compared with patients not on the drug but on csDMARDs, was associated with a diminished incidence of common, typically viral, respiratory infections. Results were consistent based on adjusted multivariable models and matched analysis across the three outcomes analysed. To our knowledge, this is the only study that examined the incidence of infections in a large number of patients receiving chronic HCQ treatment over prolonged treatment intervals compared with patients on other csDMARDs in the setting of a rheumatic disease.

HCQ antimicrobial effectiveness has been speculated to be present mainly against viruses like SARS-CoV-2 but also against parasites and intracellular bacteria. ${ }^{10-13}$ Although the vast majority of URIs are viral, there are likely some bacterial infections in the pneumonia data we report. This is the reason we separately examined the rates of URIs and bronchitis that are typically viral (excluding pneumonias that can be viral as well as primarily or secondarily bacterial).

Beyond the antiviral HCQ properties which may be responsible for a potential favourable effect on COVID-19, it has been speculated that the immunomodulatory effects of HCQ may interfere with the evolution 
Table 1 Baseline characteristics of patients starting hydroxychloroquine and other csDMARD* medications at time of start of drug for all starters and in the matched cohort

\begin{tabular}{|c|c|c|c|c|c|c|}
\hline & \multicolumn{3}{|l|}{ All starters } & \multicolumn{3}{|l|}{ Matched starters } \\
\hline & \multirow{2}{*}{$\begin{array}{l}\text { Hydroxychloroquine } \\
(\mathrm{N}=2270)\end{array}$} & \multicolumn{2}{|l|}{$\begin{array}{l}\text { Other csDMARD } \\
(\mathrm{N}=4522)\end{array}$} & \multirow{2}{*}{$\begin{array}{l}\text { Hydroxy-chloroquine } \\
\text { (N=1534) } \\
\text { Mean (SD) }\end{array}$} & \multicolumn{2}{|l|}{$\begin{array}{l}\text { Other csDMARD } \\
(\mathrm{N}=1534)\end{array}$} \\
\hline & & Mean (SD) & Std. Diff.† & & Mean (SD) & Std. Diff. \\
\hline Age & 60.05 (13.99) & $60.38(13.44)$ & 0.084 & $60.11(14.30)$ & $59.97(13.69)$ & 0.010 \\
\hline Duration of RA $\ddagger$ & $5.66 \pm 8.00$ & $5.45 \pm 8.36$ & 0.025 & $5.5 \pm 8.27$ & $5.51 \pm 7.77$ & 0.002 \\
\hline CDAI§ & $12.7 \pm 10.80$ & $17.15 \pm 13.77$ & 0.359 & $13.38 \pm 11.24$ & $13.54 \pm 11.37$ & 0.014 \\
\hline \multirow[t]{2}{*}{ BMI } & $29.53 \pm 6.96$ & $29.84 \pm 6.94$ & 0.045 & $29.54 \pm 6.96$ & $29.77 \pm 7.03$ & 0.033 \\
\hline & $\mathrm{N}(\%)$ & $\mathrm{N}(\%)$ & & $\mathrm{N}(\%)$ & $\mathrm{N}(\%)$ & \\
\hline Gender & & & 0.077 & & & 0.012 \\
\hline Men & 523 (23.2) & $1193(26.5)$ & & $368(24)$ & $360(23.5)$ & \\
\hline Women & $1730(76.8)$ & $3303(73.5)$ & & $1166(76)$ & $1174(76.5)$ & \\
\hline Hx of CVD & $311(13.7)$ & $563(12.5)$ & 0.037 & $212(13.8)$ & $216(14.1)$ & 0.008 \\
\hline Hx of Diabetes & $228(10)$ & $495(10.9)$ & 0.029 & $156(10.2)$ & $162(10.6)$ & 0.013 \\
\hline Smoking Status & & & 0.004 & & & 0.011 \\
\hline Never & $1146(51.1)$ & $2256(50.6)$ & & 795 (52.2) & 783 (51.3) & \\
\hline Previous & $723(32.3)$ & $1478(33.1)$ & & $478(31.4)$ & 491 (32.2) & \\
\hline Current & $372(16.6)$ & $728(16.3)$ & & $251(16.5)$ & $251(16.5)$ & \\
\hline $\begin{array}{l}\text { Prednisone } \\
\text { Dosage }\end{array}$ & & & 0.184 & & & 0.013 \\
\hline None & $1665(74)$ & $3040(68.4)$ & & $1112(72.5)$ & $1113(72.6)$ & \\
\hline$<7.5 \mathrm{mg}$ & $421(18.7)$ & 780 (17.5) & & $278(18.1)$ & $289(18.8)$ & \\
\hline$\geq 7.5 \mathrm{mg}$ & $165(7.3)$ & $626(14.1)$ & & $144(9.4)$ & $132(8.6)$ & \\
\hline $\begin{array}{l}\text { Number } \\
\text { csDMARD }\end{array}$ & & & 0.884 & & & 0.022 \\
\hline One & $1083(47.7)$ & 3878 (85.8) & & 973 (63.4) & 989 (64.5) & \\
\hline $2+$ & 1187 (52.3) & 644 (14.2) & & 561 (36.6) & 545 (35.5) & \\
\hline $\begin{array}{l}\text { Hx of Serious } \\
\text { Infection }\end{array}$ & $156(6.9)$ & 254 (5.6) & 0.052 & 105 (6.8) & $97(6.3)$ & 0.021 \\
\hline
\end{tabular}

${ }^{*}$ CsDMARD medications refers to conventional disease-modifying antirheumatic drugs.

†Std. Diff. is the standardised difference between the two treatment groups.

$\ddagger$ Duration of RA refers to duration of rheumatoid arthritis since initial diagnosis.

$\S C D A I$ refers to Clinical Disease Activity Index-a clinical measurement of RA disease severity.

$\eta \mathrm{Hx}$ of Serious Infection refers to history of serious upper respiratory infection, bronchitis or pneumonia prior to starting hydroxychloroquine or csDMARD medication.

of the cytokine storm which is considered to be responsible for the progression to a more serious infection. ${ }^{14} 15$ Our secondary analysis focusing only on serious respiratory infection did not identify a protective signal for serious infections in patients on HCQ.

The absence of any signal indicating that HCQ could indeed be associated with a diminished rate of these infections is potentially relevant to the present healthcare environment. Of course, it is unknown whether the dynamics of HCQ interaction with the many viruses that cause common URIs, bronchitis or pneumonia are indeed representative of its effect on the SARS-CoV-2 virus. HCQ and chloroquine do have in vitro activity against SARS-CoV-2 and a number of other respiratory viruses. Despite this, prior human studies in Dengue, influenza A and other RNA viral infections have failed to find protective effects for these compounds. ${ }^{12} 1617 \mathrm{In}$ vitro, HCQ has recently been shown to be $3 \chi$ more active than chloroquine against SARS-CoV-2 virus. ${ }^{4}$ There are limited human data as to whether this antiviral effect is present in humans. ${ }^{1256}$

It is of note that prior to matching, patients receiving HCQ monotherapy had less active disease (table 1) than patients on other csDMARDs (clinical disease activity index (CDAI) 12.7 vs $17.5, \mathrm{p}<0.001$, respectively). In addition, it would be expected that patients on the csDMARDs MTX and leflunomide (56.9\% of total csDMARDs prior to matching) would have a greater susceptibility to infections due to possible immunosuppression from these agents not seen with HCQ. In spite of these differences 
Table 2 Estimated rates of respiratory infections and estimated HRs comparing risk of infection in hydroxychloroquine compared to csDMARD* (without hydroxychloroquine use)

URI, bronchitis, pneumonia

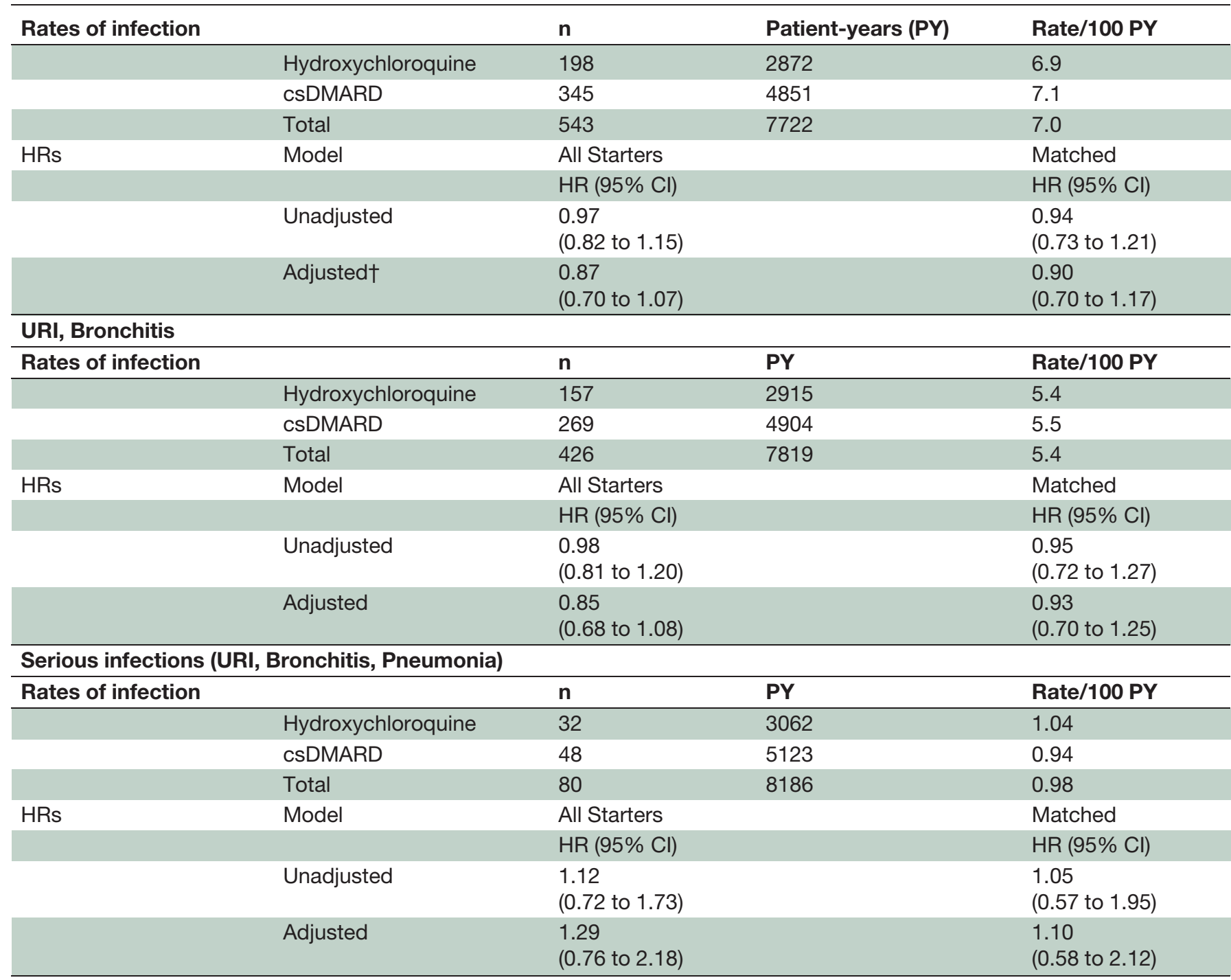

${ }^{*}$ CSDMARD medications refers to conventional disease-modifying antirheumatic drugs.

†Cox regression models adjusted for baseline characteristics and time-varying covariates including disease activity as measured by the Clinical Disease Activity Index (CDAl), prior use of 1 versus 2 or more csDMARDs, use of prednisone and its dose, season of the year, history of diabetes and history of cardiovascular disease.

that could bias towards less infection in patients on HCQ, we did not observe this effect.

Our approach has several strengths. We examined results both in the larger population of all starters with statistical adjustment and alternatively in a matched population with similar results. To our knowledge, this study is the only examination of the possible real-world protective effects of HCQ for common respiratory infections. Our study was conducted in a large population over a prolonged period of observation with systematic recording of all infections including those that occurred in the interval between visits as well as at the time of the clinical evaluation with simultaneous evaluation of important covariates such as other comorbidities and disease activity.
There are several limitations to this study. We studied patients with RA and it is possible that the effect of HCQ in this population may not be applicable to the general population who are susceptible to SARS-CoV-2. The mean age (60 years old) of the patients in the Corrona registry is perhaps greater than the mean age in the general population susceptible to this infection although the virus is of course more virulent in the older population represented in this registry. The secondary analysis of serious infections had limited number of events $(n=80)$. It is important to note that the process of recording of respiratory infections at the time of a Corrona encounter did not allow for the possible identification of a more mild or shorter duration of infection than might have occurred if the patient was not on HCQ. It is therefore not possible to rule out 


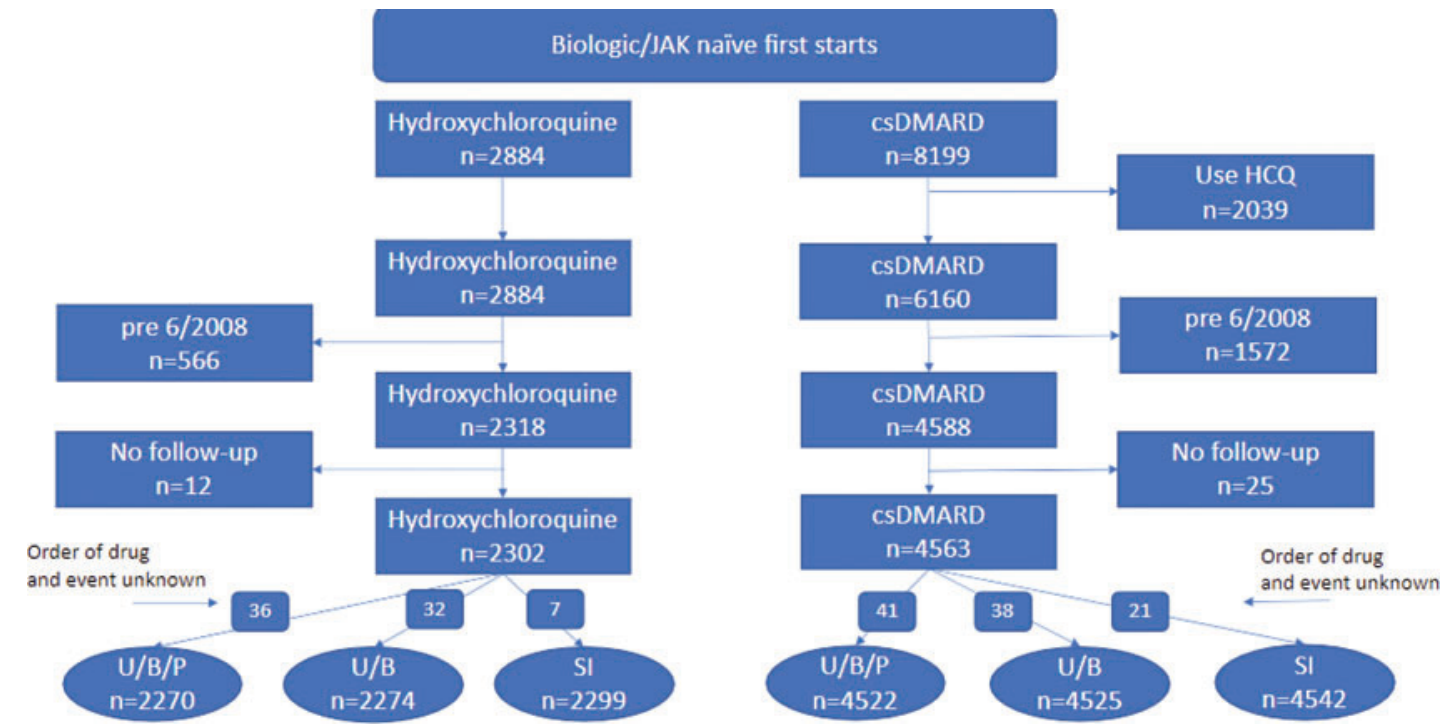

Figure 2 Kaplan-Meier curve for the primary outcome of URI, bronchitis and pneumonia.

a possible salutary effect of HCQ on the intensity or duration of viral symptoms. In addition, because we found no effect of HCQ on these infections in our database does not rule out the possibility that HCQ could be effective for the SARS-CoV-19 virus. Finally, it is possible that some infections were missed, not reported or forgotten at the time of the clinic visit when the Corrona forms were completed. Nevertheless, we believe that it is unlikely that the distribution of possible under-reporting would be skewed between the cohorts studied.

It is clear that this observational study should not be considered definitive as only an appropriately powered, prospective intervention of hydroxychloroquine in a controlled trial of patients with newly diagnosed SARSCoV-2 infection can provide reliable evidence. HCQ does not appear to decrease the risk of viral infections when compared to csDMARDs.

In summary, we found little evidence in a large number of patients with RA on HCQ that there was a diminution in respiratory infections including URIs, bronchitis or pneumonia. It is acknowledged that this experience may not necessarily be relevant to the effect of HCQ on SARSCoV-2. The effects of HCQ in patients with SARS-CoV-2 virus and COVID-19 infection should be studied in RCTs and later population-based fashion to determine if the in vitro and small blinded studies are indicative of its clinical effects in this infection. Understanding how HCQ contributes to risk in other viral infections, and respiratory infections in general, is useful as it will help inform these future investigations. It should be noted that the evidence we describe would not support the diversion of limited supplies of HCQ to the adjunctive treatment of viral syndromes in general given the reports of the challenges that many patients with both RA and SLE are having in accessing this drug needed for their routine care and well-being.
Author affiliations

${ }^{1}$ Medicine Rheumatology, Albany Medical College, Albany, New York, USA

${ }^{2}$ Orthopedics, University of Massachusetts Medical School, Worcester,

Massachusetts, USA

${ }^{3}$ Corrona, LLC, Waktham, Massachusetts, USA

${ }^{4}$ Medicine, Columbia University Medical Center, New York City, New York, USA

${ }^{5}$ Medicine, University of Massachusetts Medical School, Worcester, Massachusetts, USA

${ }^{6}$ Biostatistics, University of Massachusetts Medical School, Worcester, Massachusetts, USA

${ }^{7}$ New York University Medical Center, New York City, New York, USA

${ }^{8}$ Oregon Health \& Science University School of Medicine, Portland, Oregon, USA

Contributors All authors have contributed appropriately to qualify as authors and all authors have approved the final version of this resubmission manuscript.

Funding This study was funded by the Corrona Research Foundation, a not for profit charitable US foundation with 501(c)3 US tax status.

Competing interests JMK has received research grants in the last year from AbbVie, BMS, Gilead, Lilly, Novartis and Pfizer. He has consulted for AbbVie, BMS, Lilly, Novartis, Pfizer, Regeneron and Sanofi. He has an equity interest in Corrona, LLC and consults for them; GWR is a consultant to the Corrona Research Foundation; Dimitrios A Pappas is employed by Corrona, LLC, has received consulting honoraria from Novartis, Sanofi/Regeneron and Roche Genentech and has an equity interest in Corrona LLC. He serves on the Board of Directors of the Corrona Research Foundation; LRH is employed by Corrona and has an equity interest. She has received research grants from Pfizer and has consulted with AbbVie, BMS and Roche. She serves on the Board of Directors of the CRF; JDG is employed by Corrona, LLC and has an equity interest. He is on the Board of Directors of the CRF; KLW is a consultant for AbbVie, BMS, Galapagos, Gilead, GSK, Lilly, Pfizer and UCB. He has received research support from BMS and Pfizer.

Patient consent for publication Not required.

Provenance and peer review Not commissioned; externally peer reviewed.

Data availability statement All data relevant to the study are included in the article.

Open access This is an open access article distributed in accordance with the Creative Commons Attribution Non Commercial (CC BY-NC 4.0) license, which permits others to distribute, remix, adapt, build upon this work non-commercially, and license their derivative works on different terms, provided the original work is properly cited, appropriate credit is given, any changes made indicated, and the use is non-commercial. See: http://creativecommons.org/licenses/by-nc/4.0/. 
ORCID iD

Joel M Kremer http://orcid.org/0000-0001-6674-9901

\section{REFERENCES}

1 Gautret $\mathrm{P}$, Lagier JC, Parola $\mathrm{P}$, et al. Hydroxychloroquine and azithromycin as a treatment of COVID-19: results of an open-label non-randomized clinical trial. Int J Antimicrob Agents 2020;105949.

2 Jm Dc M, Goff JL, Mela-Lima B, et al. No evidence of rapid antiviral clearance or clinical benefit with the combination of hydroxychloroquine and azithromycin in patients with severe COVID-19 infection. Médecine Et Maladies Infectieuses 2020;2020.

3 Liu J, Cao R, Xu M, et al. Hydroxychloroquine, a less toxic derivative of chloroquine, is effective in inhibiting SARS-CoV-2 infection in vitro. Cell Discov 2020;6:16.

4 Yao X, Ye F, Zhang M, et al. In vitro antiviral activity and projection of optimized dosing design of hydroxychloroquine for the treatment of severe acute respiratory syndrome coronavirus 2 (SARS-CoV-2). Clin Infect Dis 2020;71:732-9.

$5 \mathrm{ZHj}$ C, Zhang Z, Jlang S, et al. Efficacy of hydroxychloroquine in patients with COVID-19: results of a randomized clinical trial medRxiv2020 (non-peer reviewed). Available https://www.medrxiv. org/content/10.1101/2020.03.22.20040758v2.full.pdf

6 CHEN Jun LD, LIU L, Ping LIU, et al. A pilot study of hydroxychloroquine in treatment of patients with common coronavirus disease-19 (COVID-19). J Zhejiang Univ (Med Sci) 2020;49.

7 Novartis commits to donate up to 130 million doses of hydroxychloroquine to support the global COVID-19 pandemic response sandoz.com2020. Available https://www.sandoz.com/news/ media-releases/novartis-commits-donate-130-million-doseshydroxychloroquine-support-global

8 Rogers WH. Regression standard errors in clustered samples. Stata Press: Stata Technical Bulletin Stata Press, 1993: 19-23.

9 Kremer JM. The Corrona US Registry of Rheumatic and Autoimmune Diseases. Clin Exp Rheumatol 2016;34:S96-S9.

10 Raoult D, Houpikian P, Tissot Dupont $\mathrm{H}$, et al. Treatment of $\mathrm{Q}$ fever endocarditis: comparison of 2 regimens containing doxycycline and ofloxacin or hydroxychloroquine. Arch Intern Med 1999;159:167-73.

11 Al-Bari MA. Chloroquine analogues in drug discovery: new directions of uses, mechanisms of actions and toxic manifestations from malaria to multifarious diseases. J Antimicrob Chemother 2015;70:1608-21.

12 Devaux CA, Rolain JM, Colson P, et al. New insights on the antiviral effects of chloroquine against coronavirus: what to expect for COVID19? Int J Antimicrob Agents 2020;105938.

13 Savarino A, Boelaert JR, Cassone A, et al. Effects of chloroquine on viral infections: an old drug against today's diseases? Lancet Infect Dis 2003;3:722-7.

14 Fox RI. Mechanism of action of hydroxychloroquine as an antirheumatic drug. Semin Arthritis Rheum 1993;23:82-91.

15 Chen G, Wu D, Guo W, et al. Clinical and immunologic features in severe and moderate coronavirus disease 2019. J Clin Invest 2020;130:2620-9.

16 Paton $\mathrm{NI}$, Lee L, Xu Y, et al. Chloroquine for influenza prevention: a randomised, double-blind, placebo controlled trial. Lancet Infect Dis 2011:11:677-83.

17 Touret F, de Lamballerie X. Of chloroquine and COVID-19. Antiviral Res 2020;177:104762. 